\title{
Synchrotron X-Ray Absorption Spectroscopy Study of Self-Assembled Nanoparticles Synthesized from Fe(acac) $)_{3}$ and Pt(acac) 2
}

\author{
K. Chokprasombat, ${ }^{1}$ C. Sirisathitkul, ${ }^{1}$ P. Harding, ${ }^{1}$ S. Chandarak, ${ }^{2}$ and R. Yimnirun ${ }^{3}$ \\ ${ }^{1}$ Molecular Technology Research Unit, School of Science, Walailak University, Nakhon Si Thammarat 80161, Thailand \\ ${ }^{2}$ School of Ceramic Engineering, Institute of Engineering, Suranaree University of Technology, \\ Nakhon Ratchasima 30000, Thailand \\ ${ }^{3}$ School of Physics, Institute of Science, Suranaree University of Technology and Synchrotron Light Research Institute \\ (Public Organization), Nakhon Ratchasima 30000, Thailand \\ Correspondence should be addressed to C. Sirisathitkul, schitnar@wu.ac.th
}

Received 30 May 2011; Accepted 1 July 2011

Academic Editor: Linbao Luo

Copyright $\odot 2012 \mathrm{~K}$. Chokprasombat et al. This is an open access article distributed under the Creative Commons Attribution License, which permits unrestricted use, distribution, and reproduction in any medium, provided the original work is properly cited.

The synchrotron X-ray absorption technique was used to complement electron microscopy in the investigation of nanoparticles synthesized from the coreduction of iron acetylacetonate, $\mathrm{Fe}(\mathrm{acac})_{3}$ and platinum acetylacetonate, $\mathrm{Pt}(\mathrm{acac})_{2}$. A much higher $\mathrm{Pt}$ composition than Fe leads to an extended X-ray absorption fine structure (EXAFS) spectrum for the sample that differs from that of fcc FePt nanoparticles. Most importantly, X-ray absorption near-edge structure (XANES) spectra clearly indicate the existence of $\alpha-\mathrm{Fe}_{2} \mathrm{O}_{3}$ and Pt metal. Since these monodisperse nanoparticles have a diameter of around $4 \mathrm{~nm}$ and tend to self-assemble into hexagonal arrangements, they can be modeled as Pt-rich cores with an $\alpha-\mathrm{Fe}_{2} \mathrm{O}_{3}$ shell stabilized by organic surfactants.

\section{Introduction}

Iron-platinum $(\mathrm{FePt})$ nanoparticles are a prime candidate for the next generation of ultrahigh density recording materials [1]. Whereas other magnetic materials become hysteresisfree superparamagnetic particles when their sizes are below $10 \mathrm{~nm}$, FePt nanoparticles of these sizes still exhibit ferromagnetism which is a requirement for nonvolatile recording [2]. To obtain substantial magnetic anisotropy for recording applications, not only the size but also the composition and local structure have to be controlled. It has been shown that as-synthesized FePt exhibits a chemically disordered fcc phase and is superparamagnetic but can be transformed into the ferromagnetic fct structure after heat treatment. In this $\mathrm{fct}$ arrangement, $\mathrm{Fe}$ and $\mathrm{Pt}$ atoms are in alternate planes with a balanced atomic ratio [3].

The synthesis of FePt nanoparticles conventionally uses the thermal decomposition of iron pentacarbonyl, $\mathrm{Fe}(\mathrm{CO})_{5}$ [3]. Since this starting material is very toxic, the coreduction of iron acetylacetonate, $\mathrm{Fe}(\mathrm{acac})_{3}$ and platinum acetylacetonate, $\mathrm{Pt}(\mathrm{acac})_{2}$ has been studied as a green alternative [4-7]. Metal acetylacetonates are universally regarded as versatile and nontoxic precursors in the synthesis of transition metal oxide nanoparticles [8]. However, the control of the composition and local structure of the FePt product from these starting materials still remain the subject of study. Commonly, the phase is identified by X-ray diffraction (XRD). The local composition can be obtained by energy dispersive spectroscopy (EDS) whereas the global composition is averaged by inductively coupled plasma-optical emission spectroscopy (ICP-OES).

In the case of the nanoparticles synthesized from the reaction between $\mathrm{Fe}(\mathrm{acac})_{3}$ and $\mathrm{Pt}(\mathrm{acac})_{2}$ and stabilized by organic surfactants, several possible core-shell structures may be obtained. The configuration gets more complicated when the ratio between Fe and Pt is highly unbalanced or a significant amount of oxygen is present. As a result, the conclusion about the local structure cannot be reached solely 
by using conventional characterization techniques. X-ray absorption spectroscopy offers a unique opportunity to shine light on local structures and complement the information on composition and phase. From X-ray absorption spectra, Shinoda et al. concluded that different synthetic conditions led to nanoparticles of varying structures including a Pt-rich FePt core with an Fe-rich amorphous shell [9]. Huang et al. studied the shift in absorption peaks as a function of thermal treatments [10] and copper additions [11]. In addition to Xray absorption studies of FePt, Antoniak et al. demonstrated that the technique was also useful in the case of iron oxide [12]. Since we are interested in using X-ray absorption from a synchrotron radiation source to probe such nanoparticles, self-assembled nanoparticles from metal acetylacetonate precursors are used as the case study in this work. Similar studies could then be extended to other related structures including those iron oxide-coated Pt nanoparticles [13].

\section{Experimental}

2.1. General. $\mathrm{Fe}(\mathrm{acac})_{3}(99.99 \%), \mathrm{Pt}(\mathrm{acac})_{2}$ (97\%), oleic acid (90\%), oleylamine (70\%) were obtained from Fluka Chemical Company and used as received. Benzyl ether was degassed for 15 min before use. Other AR grade organic solvents used for purification (e.g., hexane and absolute ethanol) were used as purchased. All manipulations were performed under dry nitrogen $\left(\mathrm{N}_{2}\right)$ using standard Schlenk line techniques.

2.2. Nanoparticles Preparation. A mixture of $0.5 \mathrm{mmoL}$ $\mathrm{Pt}(\mathrm{acac})_{2}$ and $0.5 \mathrm{mmoL} \mathrm{Fe}(\mathrm{acac})_{3}$ was added in a $100 \mathrm{~mL}$ Schlenk flask filled with $20 \mathrm{~mL}$ benzyl ether. Oxygen was removed from the reaction flask in vacuo before being filled with $\mathrm{N}_{2}$. Once the solution reached $120^{\circ} \mathrm{C}, 5.0 \mathrm{mmoL}$ oleic acid and $5.0 \mathrm{mmoL}$ oleylamine as surfactants were added, and the solution was then heated to $210^{\circ} \mathrm{C}$ and kept at that temperature for $30 \mathrm{~min}$. The black solution was refluxed at $300^{\circ} \mathrm{C}$ for $30 \mathrm{~min}$ then cooled to room temperature under $\mathrm{N}_{2}$. The particles were precipitated by addition of ethanol and then isolated by centrifugation. The obtained precipitate was redispersed in ethanol, followed by centrifugation. This washing procedure was repeated three times. Then, the washed particles were dispersed in hexane with a small amount (ca. $0.05 \mathrm{~mL}$ ) of oleic acid and oleylamine, followed by bubbling with $\mathrm{N}_{2}$ to remove $\mathrm{O}_{2}$. The colloid was stored in glass bottles in a refrigerator at $4^{\circ} \mathrm{C}$.

2.3. Characterization Methods. For characterization, samples were prepared by depositing the colloid on solid substrates and evaporating the solvent at room temperature. The morphology of the products was examined by transmission electron microscopy (TEM) and the elemental composition was probed by EDS. X-ray absorption spectroscopy was obtained using synchrotron radiation at BL-8, Synchrotron Light Research Institute, Thailand. The X-ray absorption nearedge structure (XANES) and extended X-ray absorption fine structure (EXAFS) measurements of the Fe $K$-edge and $\mathrm{Pt}$ $M_{5}$-edge were performed in the transmission mode with an electron energy of $1.2 \mathrm{GeV}$. The spectra were collected

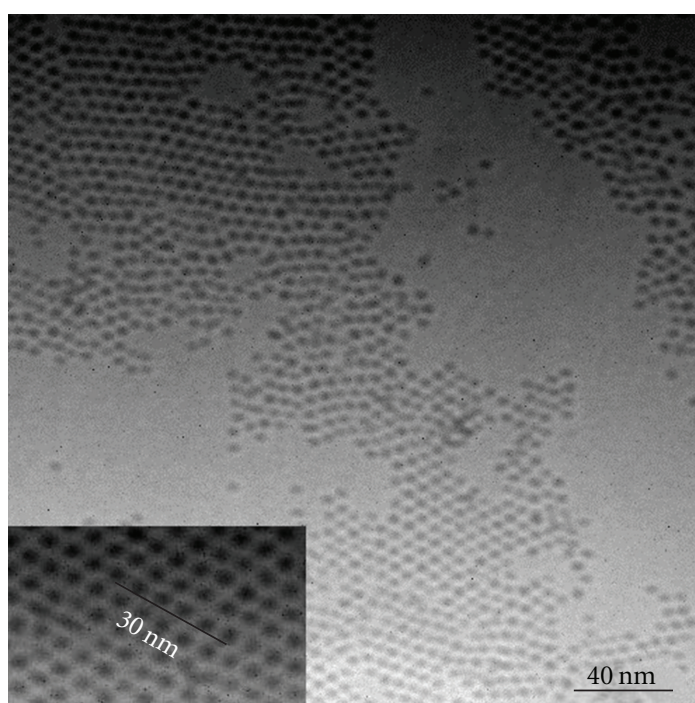

FIgURE 1: TEM images of synthesized nanoparticles.

at ambient temperature with a germanium(111) double crystal monochromator and recorded after performing an energy calibration. To increase the count rate, the ionization chamber was filled with argon gas. The storage ring was running at an energy of $1.22 \mathrm{GeV}$ with electron currents between $140 \mathrm{~mA}$ and $80 \mathrm{~mA}$.

\section{Results and Discussion}

The as-synthesized spherical nanoparticles in Figure 1 show a tendency to self-assemble into a hexagonal pattern. The higher magnification image in the inset reveals that these monodisperse particles have an approximate diameter of $4 \mathrm{~nm}$ and interparticle spacing is around $2 \mathrm{~nm}$. This agrees to the observation by Nakaya et al. that such metal core dimensions and surfactants lead to a hexagonal assembly whereas larger nanoparticles (around $6 \mathrm{~nm}$ ) tend to selfassemble into square patterns [5]. Elemental compositions of the colloid deposits by EDS analysis are 74.93\% Pt, 8.24\% $\mathrm{Fe}$, and $16.83 \% \mathrm{O}$. Even though the molar ratio of $\mathrm{Fe}: \mathrm{Pt}$ sources is $1: 1$ and a high boiling point solvent combines with high surfactants: metal ratio, $\mathrm{Pt}$ rich nanoparticles are still obtained rather than an ideal 1:1 stoichiometric FePt nanoparticles. This can be understood by the heterocoagulation model recently proposed by Beck et al. for the reaction between metal acetylacetonates [14]. In contrast to the binary nucleation model in which Fe-rich particles from the decomposition of $\mathrm{Fe}(\mathrm{CO})_{5}$ occur simultaneously with Pt-rich particles [3], $\mathrm{Fe}(\mathrm{acac})_{3}$ is harder to reduce than $\mathrm{Pt}(\mathrm{acac})_{2}$, and this leads to the intermediate products of Ptrich nuclei with the deposition of iron oxide on their surface. These iron oxides are then reduced to Fe atoms by a COspillover process on surface of the Pt nuclei, and the $\mathrm{Fe}$ atoms diffuse into the Pt-rich nuclei at high temperatures. It follows that the reduction of iron oxides is more pronounced in the case of smaller Pt nuclei (larger surface area). The composition of nanoparticles from this mechanism is 


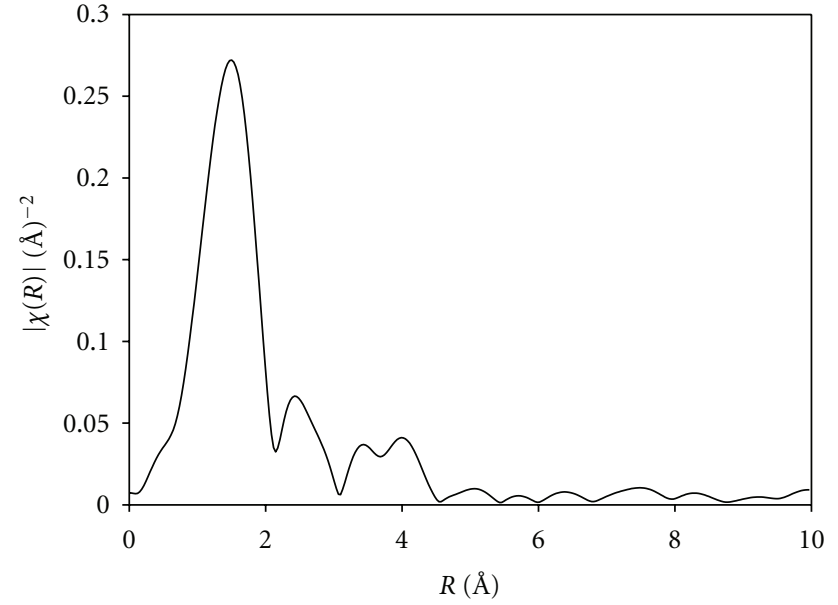

FIgURE 2: Fourier transform of Fe $K$-edge EXAFS spectrum of nanoparticles.

then sensitive to the reflux conditions as well as the amount of surfactants. Excessive amount of surfactants leads to particles of larger size but lowers the effectiveness of the reduction process. Moreover, the excess surfactants may impede the reduction process on the particle surface.

Fourier transformation of the EXAFS spectrum of the Fe $K$-edge is shown in Figure 2. The highest peak between 0.1 and $0.2 \mathrm{~nm}$ corresponds to nearest neighboring Fe-O correlation which is different from the Fe-Pt profile shown in work reported by Shinoda et al. [9]. The Fe K-edge and Pt $M_{5}$-edge XANES spectra of the nanoparticles are shown in Figure 3. Between 21002-2200 eV in Figure 3(a), the profile fits well with that of the Pt standard, indicating that the sample contains Pt metal. In contrast, the XANES spectrum between 7000 and $7200 \mathrm{eV}$ of the Fe K-edge does not match that of the Fe standard. Instead, as shown in Figure 3(b), it resembles that of hematite $\left(\alpha-\mathrm{Fe}_{2} \mathrm{O}_{3}\right)$ which is the most thermodynamically stable iron oxide at ambient conditions. XANES has the advantage of distinguishing the different valence states of $\mathrm{Fe}$ as is evident in this case because the different forms of iron oxide nanoparticles are difficult to differentiate by other techniques [12]. The results from Xray absorption spectroscopy suggest that the sample contains mostly Pt metal and $\alpha-\mathrm{Fe}_{2} \mathrm{O}_{3}$ with small amount of $\mathrm{Fe}$ metal. Since these nanoparticles have rather uniform size distribution and self-assembled arrangement, it is likely that Pt-rich cores with $\alpha-\mathrm{Fe}_{2} \mathrm{O}_{3}$ shells are formed. This coreshell structure is surrounded by oleic acid and oleylamine. Although these surfactants are not detected by TEM or X-ray absorption spectroscopy, the model is confirmed by the stability of the nanoparticles in hexane without agglomeration and sedimentation over a long period of time.

\section{Conclusion}

Self-assembled monodisperse nanoparticles were synthesized from the coreduction of $\mathrm{Fe}(\mathrm{acac})_{3}$ and $\mathrm{Pt}(\mathrm{acac})_{2}$ in the presence of benzyl ether and organic surfactants. With a composition of Fe lower than 10\%, X-ray absorption spectra are

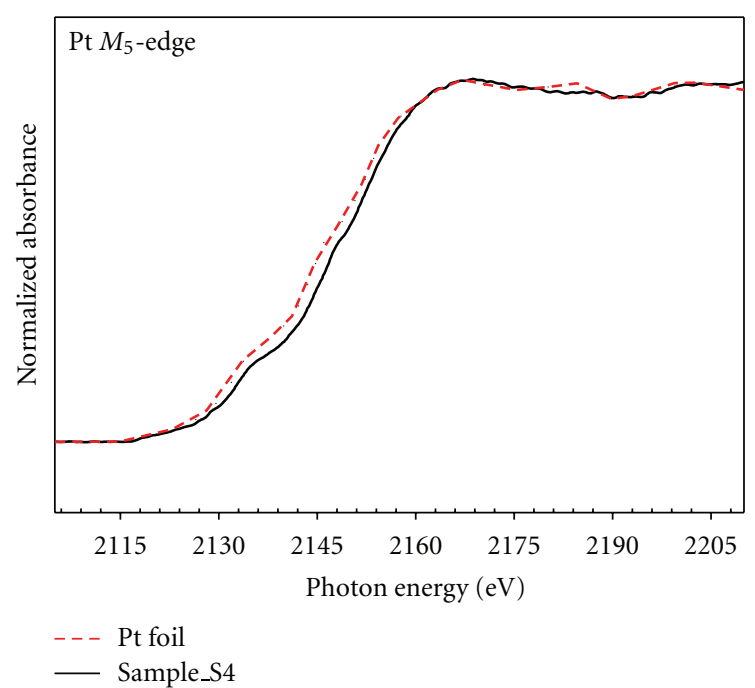

(a)

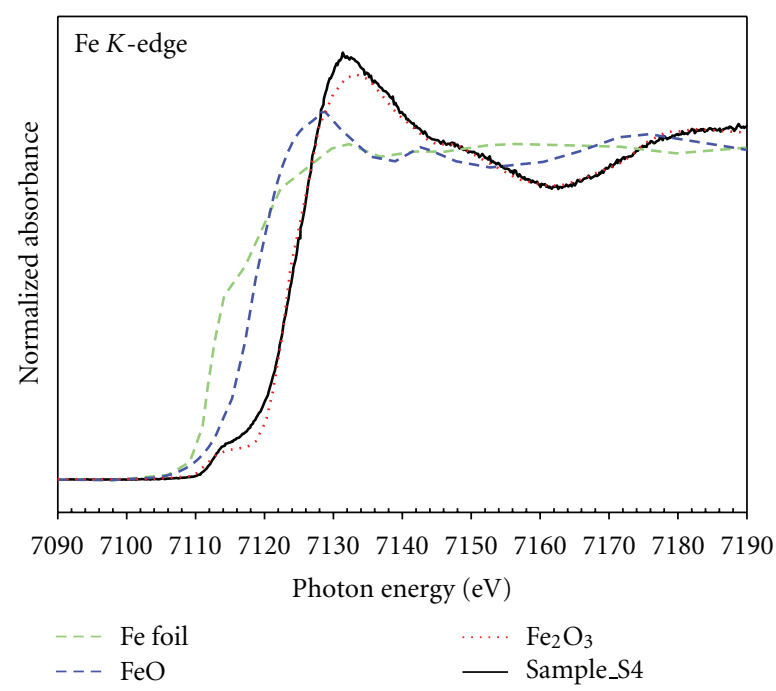

(b)

Figure 3: XANES spectra of nanoparticles (Sample_S4) for (a) Pt $\mathrm{M}_{5}$-edge compared to $\mathrm{Pt}$ foil and (b) Fe $\mathrm{K}$-edge compared to Fe foil, $\mathrm{FeO}$, and $\mathrm{Fe}_{2} \mathrm{O}_{3}$.

consistent with a Pt-rich core with a $\alpha-\mathrm{Fe}_{2} \mathrm{O}_{3}$ shell over an Fe-Pt alloy consistent with a heterocoagulation mechanism in a modified polyol process. It was demonstrated that the core shell can be modeled from the study by X-ray absorption spectroscopy and electron microscopy.

\section{Acknowledgments}

This project is financially supported by the National Electronics and Computer Technology Center, National Science and Technology Development Agency and Industry/University Cooperative Research Center (I/UCRC) in HDD Component, the Faculty of Engineering, Khon Kaen University. Support from Seagate (Thailand) and the proof-reading by Dr. D. James Harding are gratefully acknowledged. 


\section{References}

[1] B. D. Terris and T. Thomson, "Nanofabricated and selfassembled magnetic structures as data storage media," Journal of Physics D, vol. 38, no. 12, pp. R199-R222, 2005.

[2] H. W. Zhang, Y. Liu, and S. H. Sun, "Synthesis and assembly of magnetic nanoparticles for information and energy storage applications," Frontiers of Physics in China, vol. 5, no. 4, pp. 347-356, 2010.

[3] S. Sun, "Recent advances in chemical synthesis, self-assembly, and applications of FePt nanoparticles," Advanced Materials, vol. 18, no. 4, pp. 393-403, 2006.

[4] V. Nandwana, K. E. Elkins, and J. P. Liu, "Magnetic hardening in ultrafine FePt nanoparticle assembled films," Nanotechnology, vol. 16, no. 12, pp. 2823-2826, 2005.

[5] M. Nakaya, M. Kanehara, and T. Teranishi, "One-pot synthesis of large FePt nanoparticles from metal salts and their thermal stability," Langmuir, vol. 22, no. 8, pp. 3485-3487, 2006.

[6] C. Liu, X. Wu, T. Klemmer et al., "Polyol process synthesis of monodispersed FePt nanoparticles," Journal of Physical Chemistry B, vol. 108, no. 20, pp. 6121-6123, 2004.

[7] K. E. Elkins, T. S. Vedantam, J. P. Liu et al., "Ultrafine FePt nanoparticles prepared by the chemical reduction method," Nano Letters, vol. 3, no. 12, pp. 1647-1649, 2003.

[8] A. L. Willis, Z. Y. Chen, J. Q. He, Y. M. Zhu, N. J. Turro, and S. O'Brien, "Metal acetylacetonates as general precursors for the synthesis of early transition metal oxide nanomaterials," Journal of Nanomaterials, vol. 2011, Article ID 525967, 5 pages, 2011.

[9] K. Shinoda, K. Sato, B. Jeyadevan, K. Tohji, and S. Suzuki, "Local structural studies of directly synthesized L10 FePt nanoparticles by using XRD, XAS and ASAXS," Journal of Magnetism and Magnetic Materials, vol. 310, no. 2, pp. 23872389, 2007.

[10] T. W. Huang, Y. H. Huang, T. H. Tu, and C. H. Lee, "X-ray diffraction and absorption spectroscopy studies of the structure of self-assembled FePt nanoparticles during annealing," Journal of Magnetism and Magnetic Materials, vol. 282, no. SI, pp. 127-130, 2004.

[11] T. W. Huang, T. H. Tu, Y. H. Huang, C. H. Lee, and C. M. Lin, "X-ray scattering and absorption spectroscopy study of the order-disorder transition of $(\mathrm{FePt})_{1-\mathrm{x}} \mathrm{Cu}_{\mathrm{x}}$ nanoparticles," IEEE Transactions on Magnetics, vol. 41, no. 2, pp. 941-943, 2005.

[12] C. Antoniak, A. Warland, M. Darbandi et al., "X-ray absorption measurements on nanoparticle systems: self-assembled arrays and dispersions," Journal of Physics D, vol. 43, no. 47, Article ID 474007, 8 pages, 2010.

[13] H. He and C. Gao, "Synthesis of $\mathrm{Fe}_{3} \mathrm{O}_{3} / \mathrm{Pt}$ nanoparticles decorated carbon nanotubes and their use as magnetically recyclable catalysts," Journal of Nanomaterials, vol. 2011, Article ID 193510, 10 pages, 2011.

[14] W. Beck Jr., C. G. S. Souza, T. L. Silva, M. Jafelicci Jr., and L. C. Varanda, "Formation mechanism via a heterocoagulation approach of FePt nanoparticles using the modified polyol process," Journal of Physical Chemistry C, vol. 115, no. 21, pp. 10475-10482, 2011. 

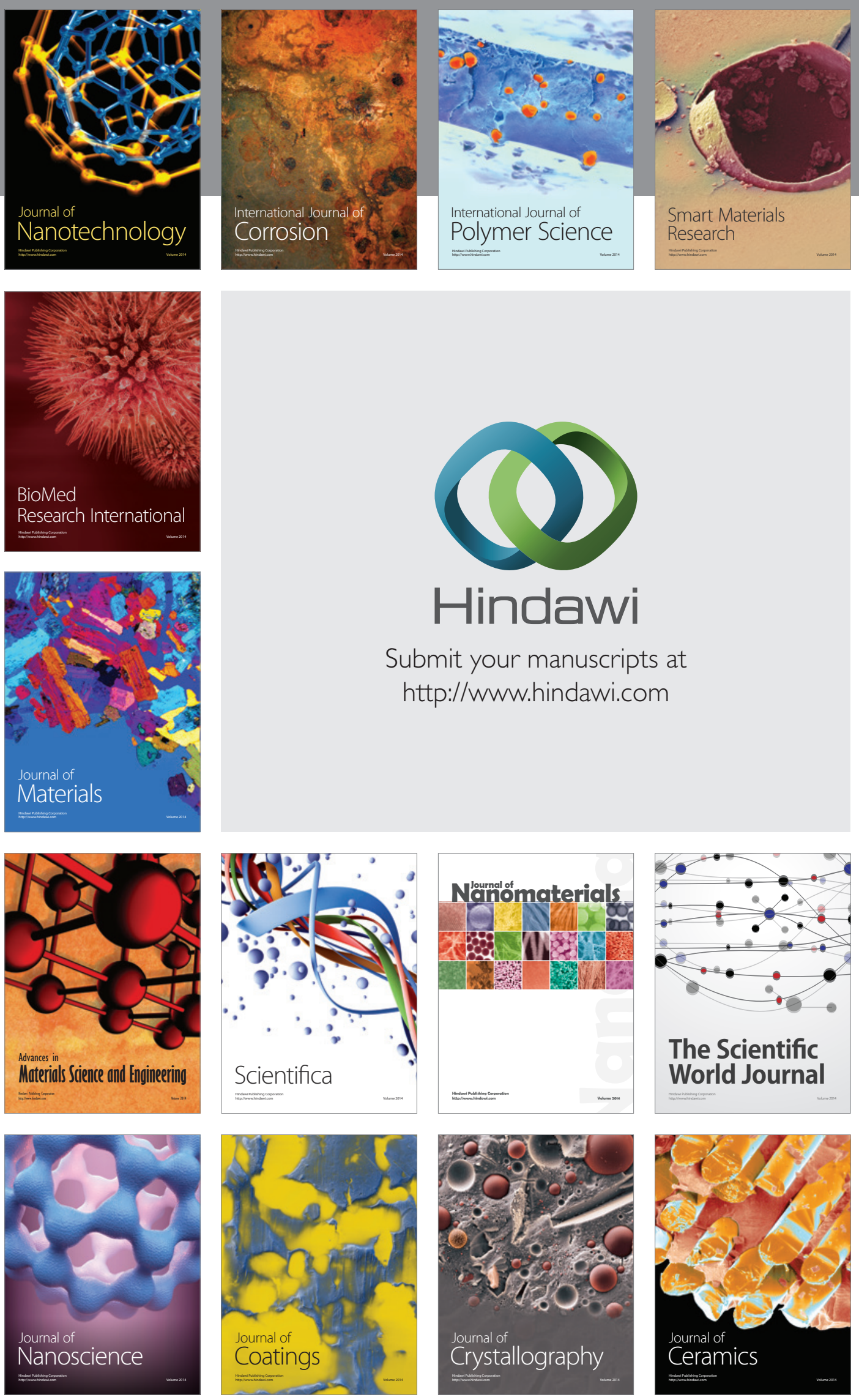

The Scientific World Journal

Submit your manuscripts at

http://www.hindawi.com

\section{World Journal}

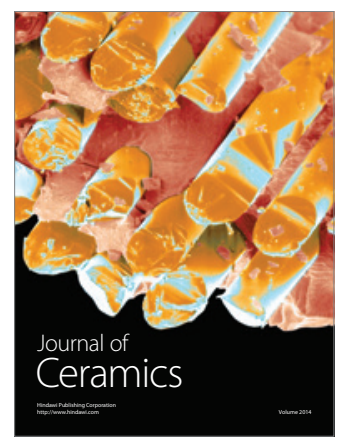

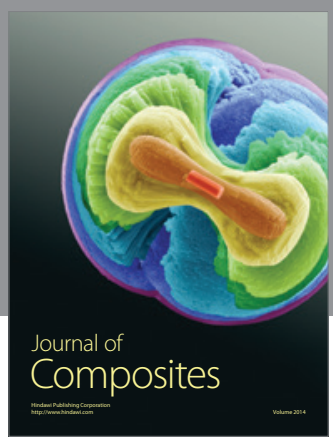
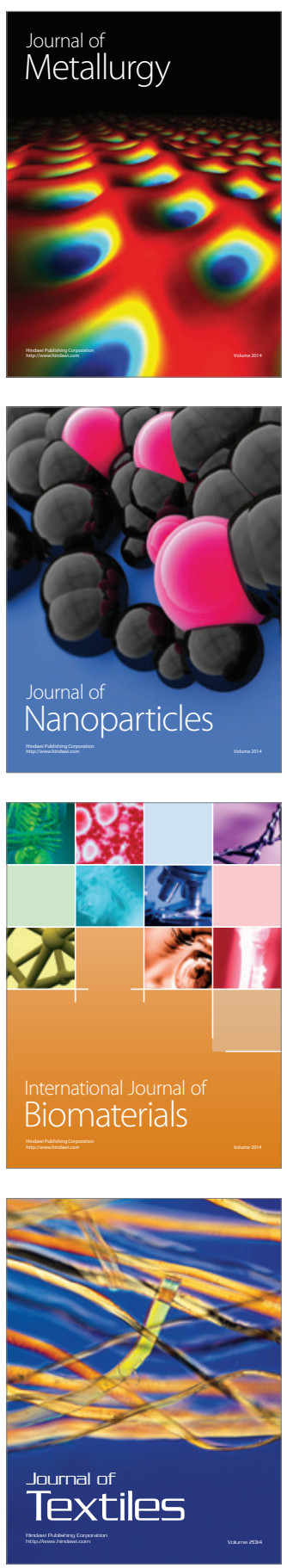